\title{
STRESS INTENSITY FACTORS CALCULATION IN ANTI-PLANE FRACTURE PROBLEM BY ORTHOGONAL INTEGRAL EXTRACTION METHOD BASED ON FEMOL *
}

\author{
Xu Yongjun \\ (Institute of Mechanics, Chinese Academy of Sciences, Beijing 100080, China) \\ Yuan Si \\ (Department of Civil Engineering, Tsinghua University, Beijing 100084, China)
}

Received 19 May 2006; revision received 30 January 2007

\begin{abstract}
For an anti-plane problem, the differential operator is self-adjoint and the corresponding eigenfunctions belong to the Hilbert space. The orthogonal property between eigenfunctions (or between the derivatives of eigenfunctions) of anti-plane problem is exploited. We developed for the first time two sets of radius-independent orthogonal integrals for extraction of stress intensity factors (SIFs), so any order SIF can be extracted based on a certain known solution of displacement (an analytic result or a numerical result). Many numerical examples based on the finite element method of lines (FEMOL) show that the present method is very powerful and efficient.
\end{abstract}

KEY WORDS anti-plane problem, Hilbert space, eigenvalue, eigenfunction, orthogonal relationship, stress intensity factor, finite element method of lines

\section{INTRODUCTION}

The stress analysis in practical engineering applications inevitably encounters stress singularities caused by sudden changes in geometry, e.g. around re-entrant corners (notches) or, more severely, around crack tips. Their presence causes great difficulty to the numerical solutions that have to be invoked when the analytical solutions are not available. From Williams ${ }^{[1--3]}$, who provided a general solution to the two-dimensional stress and displacement fields for the case of the planar crack problem, interest was mainly given to the eigenfunction expansion method. Hartranft and $\mathrm{Sih}^{[4]}$ used eigenfunction expansions in the general solution to three-dimensional crack problems, and Liu ${ }^{[5]}$ gave eigenfunction expansions of general displacements and general stresses in the Reissner plate with crack. Most interest was focused on SIF calculation with the eigenfunction expansion method. In Refs. [6-10], the boundary collocation technique is used to calculate the SIFs, while in Refs.[11,12], the advantage of boundary integral method is taken to solve the problem of a bending beam with a notch. Stern et al. ${ }^{[13,14]}$ exploited the contour integral method based on Betti's reciprocal work. Long ${ }^{[15]}$ presented the sub-region generalized variational principle, which was extended by Long et al. ${ }^{[16,17]}$ to fracture problems. Most numerical methods make direct or indirect use of the eigensolutions available in calculating SIFs for cracks/notches

\footnotetext{
* Project supported by the National Natural Science Foundation of China (Nos. 59525813 and 19872066).
} 
so that singularity can be treated in a more efficient, accurate and reliable way. Recently, Xu and Yuan et al. ${ }^{[18--23]}$ have proposed some effective methods for accurate and reliable computation of completely real or complex eigensolutions in two-dimensional notch/crack singularities with multiple materials, arbitrary opening angles and various surface conditions. The resulting algorithm is robust and can be employed by any numerical method that makes use of singular solutions. A numerical recipe for accurate and efficient computation of stress singularity factors (SIFs) usually consists of two major ingredients, namely, a powerful numerical method for general stress analysis and a novel approach to obtaining the desired SIFs which may include a special treatment of various singularities. Instead of giving an extensive review of various existing numerical approaches, the discussion is confined to a brief introduction to the major ingredients adopted in the present papers.

From an anti-plane problem, the differential operator is self-adjoint and the corresponding eigenfunctions belong to the Hilbert space. The orthogonal property between eigenfunctions (or between the derivatives of eigenfunctions) of the anti-plane problem is exploited. According to the orthogonal relationship, we developed for the first time two sets of radius-independent orthogonal integrals for extraction of stress intensity factors (SIFs), so any order SIF can be extracted based on a certain known solution to displacement (an analytic result or a numerical result). The background numerical method employed in this paper is the finite element method of lines (FEMOL) ${ }^{[24--27]}$, which is a general-purpose semi-analytical method. With this method, a partial differential equation defined in an arbitrary domain is semi-discretized, by finite element techniques based on the energy theorems or variational principles, into a system of ordinary differential equations (ODEs) defined on straight or curved mesh lines. It can be meshed easily with a group of lines radiating from the vertex of cracks/notches for fracture problems, such as anti-plane cracks/notches of multi-materials, arbitrary opening angles and different surface conditions. At present, the resulting ODE system is solved directly and efficiently by a state-of-the-art ODE solver, e.g. COLSYS ${ }^{[28,29]}$ is exclusively adopted in the present paper. These solvers have built-in self-adaptability features so the accuracy of the ODE solutions satisfies the user pre-specified error tolerances, and have no need for re-meshing. Using these solvers, FEMOL has been proved to be a remarkable numerical method with efficient adaptability in the mesh line directions automatically built in, so its inherent semi-analytical characteristics are well preserved. Its power and versatility have been demonstrated by a series of theoretical analyses and computational applications to various linear and nonlinear problems. A general-purpose computer code FEMOL92 ${ }^{[30]}$ that is capable of static and vibration analysis of various linear elastic structures has been developed. A more detailed and systematic descriptions of FEMOL see Ref.[31].

The anti-plane problem is considered in this paper, and without loss of generality, body forces are not included and only homogeneous displacement boundary conditions are taken into account. A number of illustrative numerical examples, including bi-material notches/cracks problem, are given in the paper to show the generally excellent performance of the proposed SIFs computation method. Many numerical examples based on the finite element method of lines (FEMOL) show that the method presented in this paper is very powerful and efficient.

\section{PRELIMINARY CONSIDERATION}

Figure 1 shows an anti-plane notch with $N$ different wedged materials around the notch tip. The body forces are assumed to be negligible. $G_{n}(n=1,2, \ldots, N)$ are the shear module of the materials, $\theta_{1}$ and $\theta_{N+1}$ the two boundaries of the notch which can be either stress free (F) or displacement clamped (C). $\theta_{n}(n=2, \ldots, N)$ are the interface of two materials. The differential equation can be expressed by the stresses in the $n$-th wedged material.

$$
\frac{\partial\left(r \tau_{r z_{n}}\right)}{\partial r}+\frac{\partial \tau_{\theta z_{n}}}{\partial \theta}=0, \quad n=1,2, \ldots, N
$$

The stress-strain relations are

$$
\tau_{\theta z_{n}}=\frac{1}{r} G_{n} \frac{w_{n}}{\partial \theta}, \quad \tau_{r z_{n}}=G_{n} \frac{w_{n}}{\partial r}, \quad 1 \leq n \leq N
$$




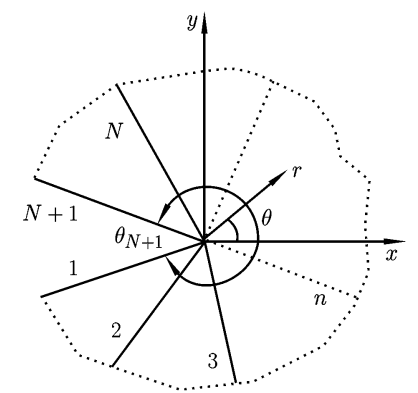

Fig. 1. $N$-material notch problem.

The equilibrium equation can be expressed by the displacement $w_{n}$ in the $n$-th wedge

$$
\frac{\partial^{2} w_{n}}{\partial r^{2}}+\frac{1}{r} \frac{\partial w_{n}}{\partial r}+\frac{1}{r^{2}} \frac{\partial^{2} w_{n}}{\partial \theta^{2}}=0, \quad n=1,2, \ldots, N
$$

It is well known that the Williams expression of $w_{n}$ can be written as

$$
w_{n}=\alpha r^{\lambda} f_{n}(\theta)
$$

Substituting $w_{n}$ into Eq.(3) yields an eigenproblem in ordinary differential equations (ODEs) as

$$
f_{n}^{\prime \prime}+\lambda^{2} f_{n}=0, \quad n=1,2, \ldots, N
$$

The boundary conditions (BCs) of notch/crack can be written as

$$
\text { F: } f_{1}^{\prime}\left(\theta_{1}\right)=0, \quad f_{N}^{\prime}\left(\theta_{N+1}\right)=0 ; \quad \text { C: } f_{1}\left(\theta_{1}\right)=0, \quad f_{N}\left(\theta_{N+1}\right)=0
$$

The displacement continuity and stress equilibrium conditions for the interface $\theta=\theta_{n}$ can be given as

$$
f_{n}\left(\theta_{n+1}\right)=f_{n+1}\left(\theta_{n+1}\right), \quad G_{n} f_{n}^{\prime}\left(\theta_{n+1}\right)=G_{n+1} f_{n+1}^{\prime}\left(\theta_{n+1}\right), \quad n=1,2, \ldots, N-1
$$

\section{EIGENFUNCTION}

To solve the eigenproblem defined by Eqs.(4)-(6), the well known explicit form of eigenfunction $f_{n}(\theta)$ can be employed, i.e.

$$
f_{n}(\theta)=A_{n} \cos (\lambda \theta)+B_{n} \sin (\lambda \theta), \quad n=1,2, \ldots, N
$$

From Eq.(6) we have the relationship with respect to $\left\{A_{n}, B_{n}\right\}$ between the two adjoining materials as

$$
\left\{\begin{array}{l}
A_{n+1} \\
B_{n+1}
\end{array}\right\}=\left[\Delta_{n}\right]\left\{\begin{array}{l}
A_{n} \\
B_{n}
\end{array}\right\}, \quad n=1,2, \ldots, N-1
$$

where

$$
\left[\Delta_{n}\right]=\left[\begin{array}{cc}
\cos \left(\lambda \theta_{n+1}\right) & -\sin \left(\lambda \theta_{n+1}\right) \\
\sin \left(\lambda \theta_{n+1}\right) & \cos \left(\lambda \theta_{n+1}\right)
\end{array}\right]\left[\begin{array}{cc}
1 & 0 \\
0 & \frac{G_{n}}{G_{n+1}}
\end{array}\right]\left[\begin{array}{cc}
\cos \left(\lambda \theta_{n+1}\right) & \sin \left(\lambda \theta_{n+1}\right) \\
-\sin \left(\lambda \theta_{n+1}\right) & \cos \left(\lambda \theta_{n+1}\right)
\end{array}\right]
$$

The matrix $\left[\Delta_{n}\right]$ is not singularity, i.e. $\left|\Delta_{n}\right|=G_{n} / G_{n+1} \neq 0$, which implies that any set of $\left\{A_{n}, B_{n}\right\}$ can be represented by other sets, namely, if we know one set of $\left\{A_{n}, B_{n}\right\}$ in the $n$-th wedge, we can calculate the others one by one from Eq.(9).

For any order eigenvalue, solving the corresponding eigenfunction $f_{n}(\theta)(n=1,2,3, \ldots, N-1)$ is equivalent to computing the coefficients $\left\{A_{n}, B_{n}\right\}$. For example, when the boundary $\theta_{1}$ is stress free (F), $A_{1}$ and $B_{1}$ are not all zero. If $\cos \left(\lambda \theta_{1}\right) \neq 0$, then $A_{1} \neq 0, A_{1}$ is normalized and we have

$$
A_{1}=1, \quad B_{1}=\frac{\sin \left(\lambda \theta_{1}\right)}{\cos \left(\lambda \theta_{1}\right)}
$$

If $\cos \left(\lambda \theta_{1}\right)=0$, then $A_{1}=0, B_{1}$ is normalized and we have

$$
A_{1}=0, \quad B_{1}=1
$$

Then we can uniquely calculate $\left\{A_{n}, B_{n}\right\}$ from Eq.(9). 


\section{ORTHOGONAL PROPERTY OF THE EIGENFUNCTIONS}

Denote

$$
f(\theta)=\left\{f_{n}(\theta) \mid \theta_{n} \leq \theta \leq \theta_{n+1}, \quad n=1,2, \ldots, N\right\}
$$

and define the inner product of any functions $f(\theta)$ and $g(\theta)$ in $C\left[\theta_{1}, \theta_{N+1}\right]$ as

$$
(f, g)^{*}=\sum_{n=1}^{N} \int_{\theta_{n}}^{\theta_{n+1}} G_{n} f_{n}(\theta) g_{n}(\theta) \mathrm{d} \theta
$$

and the induced norm as

$$
\|f\|_{2}^{*}=\sqrt{(f, f)^{*}}
$$

It is well known that $\left\{C\left[\theta_{1}, \theta_{N+1}\right],\|\cdot\|_{2}^{*}\right\}$ is bellowed to Hilbert space, denoted as $L_{2}^{*}\left[\theta_{1}, \theta_{N+1}\right]$.

Denoting the function set $\mathcal{D}(T)$ in which all functions satisfy $f(\theta) \in L_{2}^{*}\left[\theta_{1}, \theta_{N+1}\right]$, the corresponding boundary conditions (BCs) of notch/crack (5) and the interface conditions (6), and introducing the differential operator $T$ which maps $\mathcal{D}(T)$ to $L_{2}^{*}\left[\theta_{1}, \theta_{N+1}\right]$. When $f \in \mathcal{D}(T)$

$$
T f=-\mathcal{D}^{2} f(\theta)=\left\{-f_{n}(\theta) \mid \theta \in\left(\theta_{n}, \theta_{n+1}\right), n=1,2, \ldots, N\right\}
$$

By using the part integral method, it is easy to prove that $T$ is a self-adjoint and nonnegative operator.

According to Eq.(15), Eqs.(4)-(6) can be turned into solving the eigenvalue $\lambda^{2}$ and the eigenfunction $f$ of the operator $T$

$$
T f-\lambda^{2} f=0
$$

which represents a standard eigenproblem in ODEs. Denoting that the eigenspace is $M_{\lambda}=$ Span $\left\{f \mid T f=\lambda^{2} f, f \neq 0\right\}$ and the eigenvaule set is $\sigma_{p}(T)$ (which is called spectrum of $T$ ).

According to the self-adjoining operator $T$ in Hilbert space $T$, we have the following three lemmas Lemma 1. All eigenvalues $\lambda^{2}$ of $T$ are nonnegative real number.

Lemma 2. There is orthogonal property between eigenfunctions corresponding to different eigenvalues. Lemma 3. There is orthogonal property between the derivatives of the eigenfunction corresponding to different eigenvalues.

The proof of the above lemmas can be found in many literatures, such as Ref.[32].

\section{SOLUTIONS}

According to the well known Williams expression, the displacement shown in Fig.1 can be written as

$$
w=\left\{w_{n}=\sum_{i=0}^{\infty} \alpha_{i} r^{\lambda_{i}} f_{n i} \mid \theta \in\left(\theta_{n}, \theta_{n+1}\right), 1 \leq n \leq N\right\}
$$

Assuming $f_{k}$ be the eigenfunction of the corresponding eigenvalue $\lambda_{k}$,

$$
\begin{aligned}
\left(w, f_{k}\right)^{*} & =\sum_{n=1}^{N} \int_{\theta_{n}}^{\theta_{n+1}} G_{n} w_{n} f_{n k} \mathrm{~d} \theta=\sum_{n=1}^{N} \int_{\theta_{n}}^{\theta_{n+1}} G_{n} \sum_{i=0}^{\infty} \alpha_{i} r^{\lambda_{i}} f_{n i} f_{n k} \mathrm{~d} \theta \\
& =\sum_{i=0}^{\infty} \alpha_{i} r^{\lambda_{i}} \sum_{n=1}^{N} \int_{\theta_{n}}^{\theta_{n+1}} G_{n} f_{n i} f_{n k} \mathrm{~d} \theta=\sum_{i=0}^{\infty} \alpha_{i} r^{\lambda_{i}}\left(f_{i}, f_{k}\right)^{*} \\
& =\alpha_{k} r^{\lambda_{k}}\left(f_{k}, f_{k}\right)^{*}
\end{aligned}
$$

The coefficient $\alpha_{k}$ can be extracted from Eq.(18) as the following orthogonal integral equation

$$
\alpha_{k}=r^{-\lambda_{k}} \frac{\left(w, f_{k}\right)^{*}}{\left(f_{k}, f_{k}\right)^{*}}=r^{-\lambda_{k}} \frac{\sum_{n=1}^{N} \int_{\theta_{n}}^{\theta_{n+1}} G_{n} w_{n} f_{n k}}{\left(f_{k}, f_{k}\right)^{*}}, \quad k=0,1,2,3, \ldots
$$

$\alpha_{i}$ can also be thought as the expanded coefficients of a general Fourier series. 
Moreover, we can get another set of $\alpha_{k}$ based on the expansion from the derivatives of the eigenfunction as

$$
\alpha_{k}=r^{-\lambda_{k}} \frac{\left(w, \theta, f_{k, \theta}\right)^{*}}{\left(f_{k, \theta}, f_{k, \theta}\right)^{*}}=r^{-\lambda_{k}} \frac{\sum_{n=1}^{N} \int_{\theta_{n}}^{\theta_{n+1}} G_{n} w_{n, \theta} f_{n k, \theta}}{\left(f_{k, \theta}, f_{k, \theta}\right)^{*}}, \quad k=1,2,3, \ldots
$$

Difference between the above two orthogonal integral extraction equations (19) and (20) is that the constant term (namely, the zero order coefficient) has been excluded at the latter case. For any order eigenvalue, based on the displacement field calculated from a certain numerical method as the finite element method of lines (FEMOL) used in this paper, the coefficient $\alpha_{k}$ can be gotten from Eqs.(19) and (20). Furthermore, the stress intensity factors can be obtained.

\section{NUMERICAL EXAMPLES}

To assess the performance of the proposed method, several numerical examples based on the FEMOL are given in this section. The following notations are used: $r_{0}$ - radius of the circle contour, $p$ - polynomial degree used for element displacements in FEMOL, Tol - tolerance specified for ODE solutions, $E, G, v$ Young's modulus, shear modulus, Poisson's ratio.

In the subsequent examples, the singular mapping technique of FEMOL is available. Only one of the two sets of orthogonal integral extraction equations, (19) and (20), is list, which means that the numerical results are identical within the given number of digits. All of the examples are reckoned on Pentium 586-100 computer.

Example 1. Single edge crack problem

In this example, a single edge crack anti-plane problem is studied. The boundary conditions and the FEMOL meshes are shown in Fig.2. We take $p=\tilde{p}=3$ and Tol $=0.1 \%$. The computed results of coefficients $\alpha_{1}$ and $\alpha_{3}$ along different radii $r_{0}$ are tabulated in Table 1 .

Table 1. Computed results of $\alpha_{1}$ and $\alpha_{3}$

\begin{tabular}{ccc}
\hline$r_{0}$ & $\alpha_{1}\left(\lambda_{1}=0.5\right)$ & $\alpha_{3}\left(\lambda_{3}=1.5\right)$ \\
\hline 0.001 & 1.08087 & 0.33437 \\
0.1 & 1.08105 & 0.33420 \\
0.25 & 1.08108 & 0.33416 \\
0.5 & 1.08111 & 0.33413 \\
Ref.[33] & 1.081 & \\
\hline
\end{tabular}

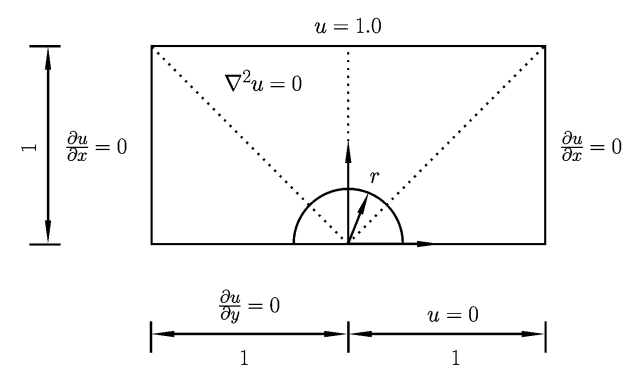

Fig. 2. Single edge crack anti-plane problem.

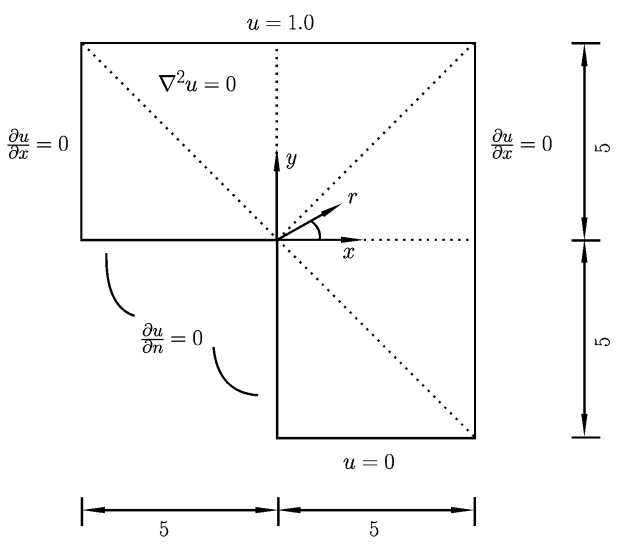

Fig. 3. L-shaped anti-plane problem.

Example 2. L-shaped anti-plane problem 
In this example, a L-shaped anti-plane problem with a notch of $90^{\circ}$ open angle is studied. The boundary conditions and the FEMOL meshes are shown in Fig. 3. We take $p=\tilde{p}=3$ and Tol=0.1\%. The well known Williams expression of displacement $u$ in this example can be written as

$$
u=\sum_{i=1,3,5, \cdots} \alpha_{i} r^{\lambda_{i}} \sin \left(\lambda_{i} \theta\right)+\sum_{j=0,2,4, \cdots} \alpha_{j} r^{\lambda_{j}} \cos \left(\lambda_{j} \theta\right)
$$

The first three lower order coefficients $\alpha_{0}, \alpha_{1}$ and $\alpha_{2}$ along different radii $r_{0}$ are computed and tabulated in Table 2 .

Table 2. Computed result of L-shape anti-plane problem

\begin{tabular}{cccc}
\hline$r_{0}$ & $\alpha_{0}\left(\lambda_{0}=0\right)$ & $\alpha_{1}\left(\lambda_{1}=2 / 3\right)$ & $\alpha_{2}\left(\lambda_{2}=4 / 3\right)$ \\
\hline 0.001 & 0.6666666667 & 0.15060 & 0.025665 \\
0.01 & 0.6666666667 & 0.15143 & 0.025400 \\
0.1 & 0.6666666669 & 0.15460 & 0.025142 \\
1.0 & 0.6666666675 & 0.15459 & 0.025137 \\
2.0 & 0.6666666672 & 0.15459 & 0.025135 \\
Best known [31] & $2 / 3$ & 0.1546 & 0.02513 \\
\hline
\end{tabular}

Example 3. Bi-material anti-plane disk with a notch

In this paper, a bi-material anti-plane disk with a right angle notch as shown in Fig.4(a) is considered. The ratio of the shear moduli of materials is taken as $G_{1}: G_{2}=10$, the open angle between two different materials is $\theta_{1}=\theta_{2}=135^{\circ}$, and the radius of the disk is taken as $R=10$.

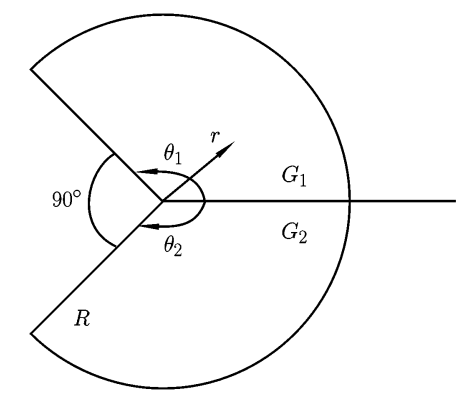

(a) bi-material anti-plane disk with a notch

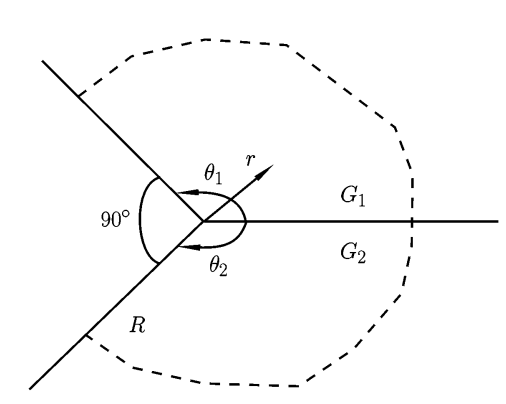

(b) bi-material in finite body with a notch

Fig. 4. Bi-material disk with a notch.

We firstly consider an infinite body with a notch shown in Fig.4(b), and the displacement is assumed to be

$$
\begin{aligned}
& w=\sum_{i=1}^{\infty} \alpha_{i} r^{\lambda_{i}} f_{i}^{n}(\theta), \quad f_{i}^{n}(\theta)=\left[a_{i}^{n} \cos \left(\lambda_{i} \theta\right)+b_{i}^{n} \sin \left(\lambda_{i} \theta\right)\right], \quad 1 \leq n \leq 2 \\
& \text { If }\left|\cos \left(\lambda_{i} \theta_{1}\right)\right| \leq\left|\sin \left(\lambda_{i} \theta_{1}\right)\right|, \quad \text { then } b_{1}^{i}=1, \quad a_{1}^{i}=\cos \left(\lambda_{i} \theta_{1}\right) / \sin \left(\lambda_{i} \theta_{1}\right) \\
& \text { If }\left|\sin \left(\lambda_{i} \theta_{1}\right)\right| \leq\left|\cos \left(\lambda_{i} \theta_{1}\right)\right|, \quad \text { then } a_{1}^{i}=1, \quad b_{1}^{i}=\sin \left(\lambda_{i} \theta_{1}\right) / \cos \left(\lambda_{i} \theta_{1}\right)
\end{aligned}
$$

The other coefficients $\left(a_{2}^{i}, b_{2}^{i}\right)$ can be calculated from the interface conditions. We take the first three terms and let $\alpha_{1}=0.01, \alpha_{2}=0.1, \alpha_{3}=1.0$, then the displacement on the circle of $r=10$ can be determined and used as the boundary condition for the disk as shown in Fig.4(a). Both of the anti-plane disk with a notch and the infinite body with a notch have the same coefficients $\alpha_{1}, \alpha_{2}, \alpha_{3}$.

We take four FEMOL elements and $p=\tilde{p}=4$ and Tol $=0.01 \%$. The first three lower order coefficients $\alpha_{1}, \alpha_{2}$ and $\alpha_{3}$ along different radii $r_{0}$ are computed and tabulated in Table 3. 
Table 3. Computed results of $\alpha_{1}, \alpha_{2}$ and $\alpha_{3}$

\begin{tabular}{cccc}
\hline$r_{0}$ & $\alpha_{1}$ & $\alpha_{2}$ & $\alpha_{3}$ \\
\hline 0.001 & 0.00999996 & 0.100003 & 1.012677 \\
0.01 & 0.00999996 & 0.100002 & 1.000452 \\
1.0 & 0.00999996 & 0.100003 & 1.000290 \\
4.0 & 0.00999996 & 0.100003 & 1.000331 \\
8.0 & 0.00999996 & 0.100003 & 1.000352 \\
exact results & 0.01 & 0.1 & 1.0 \\
\hline
\end{tabular}

\section{CONCLUSIONS}

The following conclusions can be drawn:

(1) $i$-th order SIFs directly extraction: The orthogonal relationships between eigenfunctions are exploited and two sets of orthogonal integral extraction algorithms for SIF calculations are developed. It is crucial that any order characteristic coefficient or SIF can be extracted directly.

(2) Contour integral radius independence: The two sets of orthogonal integral extraction algorithms show that the present methods are independent of the contour integral radius. A good proposal is that the radius for contour integral can not be too small or too large, because most of the methods based on stress analytical solution could not give a satisfied field in too small or too large radius domains.

(3) Self verification: The computed results can be verified by itself by the two orthogonal integral extraction algorithms and the characteristic of the independent contour integral radius.

(4) Generality: The present algorithms are general SIFs computing method and are applicable to anti-plane problem of the crack/notches with arbitrary opening angles, multiple materials and notch boundary surface conditions.

(5) Accuracy: Since the present algorithms have not led to any error, so the accuracy of the final results only relies on the based numerical methods. The FEMOL used in this paper is a semi-analytic method and is based on the ODE solver COLSYS, in which the self-adaptability is automatically built, and the accuracy is fully controlled by the user with a desired error tolerance specified to the solver.

(6) Reliability: Because of the two sets of orthogonal integral extraction relationship, the SIFs results are almost independent of the radius.

(7) Efficiency: The present algorithms only need to be integrated along an arc, so it is better to avoid too small radius or too large radius.

\section{References}

[1] Williams M. L., Stress singularities resulting from various boundary conditions in angular corners of plates in tension. J. Appl. Mech., 1952, 14: 526-528.

[2] Williams M. L., on the stress distribution at the base of a stationary crack. J. Appl. Mech. 1957, 24: 109-114.

[3] Williams M. L., Surface stress singularities resulting from various boundary conditions in angular coners of plates under bending. Proc. First U. S. Nat, Congress of Appl Mech., 1950: 325-329.

[4] Hartranft R. J. and Sih G. C., The use of eigenfunction expansions in the general solution of threedimensional crack problems. Journal of Mathematics and Mechanics, 1969, 19(2): 123-138.

[5] Liu Chuntu, Stress and deformation near the crack tip for bending plate. Acta Mechanica Solida Sinica, 1983, 3: 441-448 (in Chinese)

[6] Gross B., Srawley J. E. and Brown W. F., Stress intensity factors for a single-edge-notch tension specimen by boundary collocation, NASA TN D-2395, 1964.

[7] Kobayashi A. S., Cherepy R. B. and Kinsel W. C., A numerical procedure for estimating the stress intensity factor of a crack in a finite plate. Journal of Basic Engineering, 1964, 86: 681-684.

[8] Gross B. and Mendelson A., Plane elastic analysis of V-notched plates. Int. J. Fract. Mech., 1972, 8: $267-276$.

[9] Wilson W. K., Numerical method for determining stress intensity factors of an interior crack in a finite plate. Journal of Basic Engineering, 1971: 685-690.

[10] Carpenter W. C., A collocation procedure for determining fracture mechanics parameters at a corner. International Journal of Fracture, 1984, 24: 255-266.

[11] Rzasnicki W., Mendelson A and Albers L. U., Application of boundary integral method to elastic analysis of V-notched beams. NASA TN-F-7424, 1973.

[12] Rzasnicki W. and Mendelson A, Application of boundary integral method to elastoplastic analysis of Vnotched beams. International Journal of Fracture, 1975, 11: 329-342. 
[13] Stern M., Becker E. B. and Dunham R. S., A contour integral computation of mixed-mode stress intensity factors. International Journal of Fracture, 1976, 12(3): 359-368.

[14] Mohan Lal Soni and M. Stern, On the computation of stress intensity factors in composite media using a contour integral method. Int. J. Fract., 1976, 12(3): 331-344.

[15] Long Yuqiu, Zhi Bingchen, Kuang Wenqi, Shan Jian, Sub-region mixed finite element method for the calculation of stress intensity factor. Sinica Mechanica, 1982, 4: 341-353 (in Chinese).

[16] Long Yuqiu, Zhi Bingchen and Yuan Si, Sub-region, sub-item and sub-layer generalized variational principles in elasticity//Proceedings of International Conference on Finite Element Methods, (ed. He Guangqian and Y. K. Cheung), Shanghai, 1982: 607-609.

[17] Long Yuqiu and Zhao Yiqiang, Technical note: Calculation of stress intensity factors in plane problems by the sub-region mixed finite element method. Adv. Eng. Software, 1985, 7(1): 32-35.

[18] Xu Yongjun, Yuan Si, Complete eigensolutions for plane notches with multi-materials by the imbedding method. International Journal of Fracture, 1996, 81: 373-381.

[19] Xu Yongjun, Yuan Si, Complete eigensolutions for anti-plane notches with multi-materials by super-inverse iteration. Acta Mechanica Solida Sinica, 1997, 10(2): 157-166.

[20] Xu Yongjun, Yuan Si, Liu Chuntu, The progress on complete engen-solution of two dimensional notch problems. Advances in mechanics, 2000, 30(2): 216-226 (in Chinese).

[21] Xu Yongjun, Yuan Si, Liu Chuntu, Possible multiple roots for fracture problems. Acta Mechanica Sinica, 1999, 31(5): 618-624 (in Chinese).

[22] Xu Yongjun, Eigenproblem in fracture mechanics for reissner plate. Acta Mechanica Solida Sinica, 2004, 25(2): 225-228 (in Chinese).

[23] Xu Yongjun, Liu Chuntu, The eigenvalues and eigenfunctions in shallow shell fracture analysis. Acta Mechanica Solida Sinica, 2000, 21(3): 256-260 (in Chinese).

[24] Yuan Si, A new semi-discrete method - the finite element method of lines//Proceedings of 1st National Conference on Analytical and Numerical Combined Methods, Hunan, 1990: 132-136 (in Chinese).

[25] Yuan Si and Gao Jianling, A new computational tool in structural analysis: the finite element method of lines (FEMOL)//Proceedings of International Conference on EPMESC, Macau, 3 (Aug. 1-3, 1990): 517-526.

[26] Yuan Si, The finite element method of lines. Chinese Journal of Numerical Mathematics and Applications, 1993, 15(1): 45-59.

[27] Yuan Si, The Finite Element Method of Lines: Theory and Applications. Beijing-New York: Science Press, 1993.

[28] Ascher U., Christiansen J. and Russell R. D., Collocation software for boundary-value ODEs. ACM Transaction of Mathematical Software, 1981, 7(2): 209-222.

[29] Ascher U., Christiansen J. and Russell R. D., Algorithm 569, COLSYS: collocation software for boundaryvalue ODEs [D2]. ACM Transaction of Mathematical Software, 1981, 7(2): 223-229.

[30] Yuan Si, A general-purpose FEMOL program-FEMOL92. Computational Structural Mechanics and Applications, 1993, 10(1): 118-122 (in Chinese).

[31] Yuan Si, The finite element method of lines: theory and applications. Beijing-New York: Science Press, 1993.

[32] Erwin Kreyszig, Introductory Functional Analysis with Application. JOHN WILEY \& SONS, 1978.

[33] Xanthis L. S., A pseudo-ODE modeling trick for the direct method of lines computation of important fracture mechanics parameters. ACM SIGNUM Newsletter, 1986, 21(1-2). 